\title{
Electromagnetic Field and Wave Propagation in Gravitation*
}

\author{
Tse Chin Mo and C. H. Papas \\ Electrical Engineering Department, California Institute of Technology, Pasadena, California 91109
}

(Received 10 December 1970)

\begin{abstract}
From the physical three-vector Maxwell equations for an electromagnetic (E.M.) field in static gravitation, we examine the artifice of replacing the gravitation by an equivalent medium and we find modified Debye potentials for an E.M. wave in a simple, angularly homogeneous, material medium in a Schwarzschild gravitational field. The fact that these potentials do not obey the generalized scalar wave equation implies that gravitation scatters the vector E.M. wave and a scalar wave differently. Also, we obtain and solve by perturbation the amplitude and eikonal equations for a high-frequency wave in a weak spherical gravitational field. To the order $M / r$, the state of transverse polarization does not change along a ray path whereas the transverse-field amplitudes are modified by the factor $e^{M / r}$ which strengthens the field near the mass. The longitudinal-field amplitude, on the other hand, is modified by $e^{-M / r}$. These effects, in principle, may provide a further test of classical E.M. theory and general relativity.
\end{abstract}

\section{INTRODUCTION AND SUMMARY}

$T$ HE bending of light and electromagnetic (E.M.) waves by a spherical gravitational field has been known for many years. ${ }^{1}$ Recently, the delay in traveling time of radar signals, which appears together with the bending as a general-relativistic effect along the path of propagation, has attracted both theoretical ${ }^{2}$ and experimental ${ }^{3}$ interest. The underlying methods of analysis have made use of the photon null geodesics, or an "equivalent" index of refraction to account for the geometrical-optics effect due to gravitation.

However, except for some static problems, the dynamic gravitational influence on the amplitude and polarization of an E.M. wave has not been treated before, nor have the Debye potentials of such waves been found. ${ }^{4}$ Furthermore, the concept of replacing gravity by an "equivalent medium" was not clear. ${ }^{5}$ These problems are investigated here.

In Sec. II A, from the physical three-vector Maxwell equations for an E.M. field in static gravitation, we clarify how and in what sense an equivalent medium replaces such gravitation. In Sec. II B we express an

* This work was supported by the U. S. Air Force Office of Scientific Research, Grant No. AFOSR 70-1935.

${ }^{1}$ A. Einstein, The Meaning of Relativity (Princeton U. P., Princeton, N. J., 1955), p. 93; or see any test on relativity.

${ }^{2}$ I. I. Shapiro, Phys. Rev. Letters 13, 789 (1964); Phys. Rev. 141, 1219 (1966); D. K. Ross and L. I. Schiff, Phys. Rev. 141, 1215 (1966); D. O. Muhleman and I. D. Johnston, Phys. Rev. Letters 17, 455 (1966).

${ }^{3}$ I. I. Shapiro, G. H. Pettengill, M. E. Ash, M. L. Stone, W. B. Smith, R. P. Ingalls, and R. A. Brockelman, Phys. Rev. Letters 20, 1265 (1968); J. Anderson and P. Esposito, American Institute of Aeronautics and Astronautics Annual Meeting, 1970, Report No. 70-1317, 1970 (unpublished). For recent observations on radio bending, see D. O. Muhleman, R. D. Ekers, and E. B. Fomalont, Phys. Rev. Letters 24, 1377 (1970).

${ }^{4}$ For static problems, see, e.g., F. Occhionero and M. Demianski, Phys. Rev. Letters 23, 1128 (1969); J. L. Safko, Ann. Phys. (N. Y.) 58, 322 (1970); A. W. Martin and P. L. Pritchett, J. Math. Phys. 9, 593 (1968); also for the Friedmann universe, see P. C. Petters, J. Math. Phys. 10, 1216 (1969); for light intensities obtained by counting ray density, see S. Refsdal, in Proceedings of the International Conference on General Relativity, King's College, London, 1965, Vol. 1 (unpublished).

${ }^{5}$ C. M $\phi l l e r$, Theory of Relativity (Oxford U. P., London, 1952), p. 305 .
E.M. wave in an angularly homogeneous simple material medium in an external Schwarzschild gravitational field by its modified Debye potentials. We find that even in the vacuum limit these potentials do not satisfy the generalized scalar wave equation, and thus see that such a gravitational field scatters a vector E.M. wave and a scalar wave differently, contrary to previous belief. ${ }^{6}$

In Sec. III A we simply find the approximate amplitude and familiar eikonal equations for a high-frequency wave in a weak spherical gravity, and in Sec. III B we solve them by perturbation. To order $M / r$ we find that along a ray path the state of transverse polarization is not changed, whereas the transverse-field amplitudes are changed by $e^{M / r}$. Thus the transverse fields become stronger the nearer to the mass $M$ they get. On the other hand, the longitudinal-field amplitude, if it exists, is modified by the factor $e^{-M / r}$. Although it may not be within the present capacity of the existing NASA deepspace network to measure them, these effects in principle may provide a further test of the validity of today's general-relativistic E.M. theory. ${ }^{\text {? }}$

\section{PHYSICAL ELECTROMAGNETIC FIELD}

\section{A. In Static Gravitation}

In a static gravitational field described by a synchronous coordinate frame $\left\{x^{\mu}\right\} \equiv\left\{t, x^{1}, x^{2}, x^{3}\right\}$ with metric $g_{0_{i}} \equiv 0 \quad(i=1,2,3)$ and $g_{\mu \nu}$ not functions of time, and with the neglect of the E.M. contribution to gravitation, the Maxwell equations for physical E.M. fields are ${ }^{8}$

$$
\begin{aligned}
& \boldsymbol{\nabla}_{g} \times\left[\left(\sqrt{ } g_{00}\right) \mathbf{E}\right]=-\frac{\partial}{\partial t}-\mathbf{B}, \\
& \boldsymbol{\nabla}_{g} \cdot \mathbf{B}=0, \\
& \boldsymbol{\nabla}_{g} \times\left[\left(\sqrt{ } g_{00}\right) \mathbf{H}\right]=\left(\sqrt{ } g_{00}\right) \mathbf{J}+\frac{\partial}{\partial t} \mathbf{D}, \\
& \nabla_{g} \cdot \mathbf{D}=\rho .
\end{aligned}
$$

${ }^{6}$ R. A. Matzner, J. Math. Phys. 9, 163 (1968).

${ }^{7}$ For example, see J. L. Synge, General Relativity (Interscience, New York, 1960), p. 354.

${ }^{8}$ T. C. Mo, J. Math. Phys. 11, 2589 (1970), Sec. 4A. 
Here all fields and sources are locally physically measurable quantities in the usual sense for observers fixed in $\left\{x^{\mu}\right\}$ with $x^{i} \equiv$ const, and $\boldsymbol{\nabla}_{g}$ is the conventional del operator in the spatial coordinates $\left\{x^{i}\right\}$ with length interval $d \sigma^{2}=-g_{i j} d x^{i} d x^{j}$. The constitutive relations between $(\mathrm{D}, \mathrm{H})$ and $(\mathbf{E}, \mathrm{B})$ are determined completely by the local intrinsic physics of the medium as if there were no gravitation, except for possible gravitationally induced strains in the medium.

It must be clear that no equivalent medium can rigorously replace gravitation to account for its effect on the E.M. field. However, for linear media in which the physical fields are related by

$$
\mathrm{D}=\boldsymbol{\varepsilon} \cdot \mathrm{E}+\boldsymbol{\alpha} \cdot \mathrm{B}, \quad \mathrm{H}=\boldsymbol{\lambda} \cdot \mathrm{E}+\mathrm{K} \cdot \mathrm{B}
$$

we can define new mathematical symbols by

$$
\mathbf{e} \equiv\left(\sqrt{ } g_{00}\right) \mathbf{E}, \quad \mathbf{b} \equiv \mathbf{B}, \quad \mathbf{d} \equiv \mathbf{D}, \quad \mathbf{h} \equiv\left(\sqrt{ } g_{00}\right) \mathbf{H} .
$$

Then only for these so-defined fictitious fields $(\mathbf{e}, \mathrm{b})$, $(\mathbf{d}, \mathbf{h})$ can we replace gravitation by an "equivalent medium," using

$$
\mathbf{d}=\left[\boldsymbol{\varepsilon} /\left(\sqrt{ } g_{00}\right)\right] \cdot \mathbf{e}+\boldsymbol{\alpha} \cdot \mathbf{b}, \quad \mathbf{h}=\boldsymbol{\lambda} \cdot \mathbf{e}+\left(\sqrt{ } g_{00}\right) \mathbf{K} \cdot \mathbf{b}
$$

as "constitutive relations" and writing Maxwell's equations in curvilinear coordinates $\left\{x^{i}\right\}$ with $\boldsymbol{\nabla}_{g}$ as the differential operator and $\left(\rho,\left(\sqrt{ } g_{00}\right) \mathrm{J}\right)$ as the source. Although their difference is very simple, we must not confuse $^{5}$ these fictitious fields (3) and their constitutive relations (4) with their real physical counterparts. Except in the few calculations that field amplitudes do not occur, such as in the eikonal equation of geometric optics, ${ }^{9}$ wrongly identifying these fictitious fields with the real fields leads to incorrect results and misleading concepts.

\section{B. Debye Potentials in Spherical Gravitation}

Generally, we have to put the field equations in scalar form before solving them. For many E.M. problems in sourceless regions, the Debye potentials provide such a powerful means of doing this. ${ }^{10}$

Now, for an angularly homogeneous simple medium ${ }^{11}$ with $\varepsilon=\epsilon(\boldsymbol{r}, t) \delta_{i j}, \mathbf{K}=[\mu(\boldsymbol{r}, t)]^{-1} \delta_{i j}, \boldsymbol{\alpha}=\boldsymbol{\lambda}=0$ in spherical gravitation (e.g., a radial distribution of an isotropic stellar plasma), such potentials can still be found. First, in the standard Schwarzschild coordinates $\{t, r, \theta, \phi\}$ with metric of $d s^{2}=(1-2 M / r) d t^{2}-(1-2 M / r)^{-1} d r^{2}-r^{2} d \Omega^{2}$ for external gravitation, (1) become

$$
\begin{aligned}
\boldsymbol{\nabla} \times \mathrm{E}=-\left(1-\frac{2 M}{r}\right)^{-1 / 2} \frac{\partial \mathrm{B}}{\partial t} & \\
+ & \frac{1}{r} \frac{\partial}{\partial r}\left\{\left[\left(1-\frac{2 M}{r}\right)^{1 / 2}-1\right] \mathbf{E} \times \mathbf{r}\right\},
\end{aligned}
$$

${ }^{9} \mathrm{~V}$. Fock, The Theory of Space Time and Gravitation (Pergamon, New York, 1964), 2nd ed., p. 221.

${ }^{10}$ See, e.g., Van De Hulst, Light Scattering by Small Particles (Wiley, New York, 1957). Also, F. E. Borgnis and C. H. Papas,

$$
\begin{aligned}
\nabla \times \mathrm{H}=\mathrm{J}+(1 & \left.+\frac{2 M}{r}\right)^{-1 / 2} \frac{\partial \mathrm{D}}{\partial t} \\
& +\frac{1}{r} \frac{\partial}{\partial r}\left\{\left[\left(1-\frac{2 M}{r}\right)^{1 / 2}-1\right] \mathrm{H} \times \mathbf{r}\right\} .
\end{aligned}
$$

Here, for practical purposes we have written (5) in terms of the conventional flat 3 -space $\boldsymbol{\nabla}$ operators using the above $\{r, \theta, \phi\}$ as usual spherical coordinates. Also, the local charge-conservation law from $(5 \mathrm{~b})$ reads

$$
\begin{aligned}
-\frac{\partial}{\partial t} \rho & =\left(1-\frac{2 M}{r}\right)^{1 / 2} \\
& \times\left(\nabla \cdot \mathrm{J}+\frac{1}{r^{2}} \frac{\partial}{\partial r}\left\{r^{2} J^{(r)}\left[\left(1-\frac{2 M}{r}\right)^{1 / 2}-1\right]\right\}\right) .
\end{aligned}
$$

Then, with a familiar field decomposition ${ }^{12}$ into electric and magnetic waves having no radial magnetic and electric field, respectively, after lengthy calculation from (5) we find

$$
\begin{array}{r}
\mathbf{E}=\frac{1}{\epsilon}\left\{\left(1-\frac{2 M}{r}\right)^{1 / 2} \mathbf{U}+\left[1-\left(1-\frac{2 M}{r}\right)^{1 / 2}\right] \mathbf{e}_{r} \mathbf{e}_{r}\right\} \\
\cdot \nabla \times[\nabla \times(\mathbf{r} v)]-\left(1-\frac{2 M}{r}\right)^{1 / 2} \frac{\partial}{\partial t} \nabla \times(\mathbf{r} u), \\
\mathbf{B}=\frac{\mu}{(1-2 M / r)^{1 / 2}} \frac{\partial}{\partial t} \nabla \times(\mathbf{r} v) \\
+\left\{\left(1-\frac{2 M}{r}\right)^{1 / 2} \mathbf{U}+\left[1-\left(1-\frac{2 M}{r}\right)^{1 / 2}\right] \mathbf{e}_{r} \mathbf{e}_{r}\right\} \\
\cdot \nabla \times[\nabla \times(\mathbf{r} u)],
\end{array}
$$

where the modified electric and magnetic Debye potentials $v$ and $u$ satisfy

$$
\begin{aligned}
& \nabla^{2}\left(\begin{array}{l}
v \\
u
\end{array}\right)-\frac{1}{1-2 M / r}\left[\begin{array}{c}
\frac{\partial}{\partial t}\left(\begin{array}{c}
\partial-v \\
\partial t
\end{array}\right) \\
\mu \frac{\partial}{\partial t}\left(\begin{array}{c}
\frac{\partial}{\partial t} u \\
\partial t
\end{array}\right)
\end{array}\right]-\frac{1}{r}\left[\begin{array}{l}
\frac{\epsilon^{\prime}}{-\frac{\partial}{\partial r} r} \\
\frac{\mu^{\prime}}{\mu} \frac{\partial}{\partial r} r u
\end{array}\right] \\
& -\frac{2 M}{r}\left[\begin{array}{c}
\epsilon \frac{\partial}{\partial r}\left(\frac{1}{\epsilon r} \frac{\partial}{\partial r} r\right) \\
\mu \frac{\partial}{\partial r}\left(\frac{1}{\mu r} \frac{\partial}{\partial r} r u\right)
\end{array}\right)=0
\end{aligned}
$$

in Handbuch der Physik, edited by S. Flügge (Springer, Berlin, 1958), Vol. 16, p. 285

${ }^{11}$ For radially stratified simple medium in flat space, see $\mathrm{R}$. Latham, Can. J. Phys. 46, 1463 (1968).

${ }^{12}$ M. Born and E. Wolf, Principles of Optics (Pergamon, New York, 1970), 4th ed., p. 634 . 
and $\mathbf{U} \equiv$ unit dyadic and $\mathbf{e}_{r} \equiv$ unit radial vector. The boundary conditions ${ }^{13}$ at any radial discontinuity of $\mu, \epsilon$ are just the continuity of $v,(1 / \epsilon)(\partial / \partial r) r v, u$, and $\mu(\partial / \partial r) r u$.

We notice from (8) that even for the vacuum $\mu \equiv 1$, $\epsilon \equiv 1$, the modified Debye potentials obey

$$
\left[\nabla^{2}-\frac{1}{(1-2 M / r)} \frac{\partial^{2}}{\partial t^{2}}\right] \psi-\frac{2 M}{r} \frac{\partial}{\partial r}\left(\frac{1}{\gamma} \frac{\partial}{\partial r} r \psi\right)=0
$$

and not the generalized wave equation $\Psi_{; \mu}^{; \mu}=0$. Separating variables as usual by writing

$$
\psi \equiv e^{-i \omega t}[R(r) / r] \Theta(\theta) \Phi(\phi),
$$

with $\Phi_{m}=e^{ \pm i m \phi}, \Theta_{l m}=P_{l}{ }^{m}(\cos \theta)$, where $m=-|l|$ to $|l|$ and $l=$ integer, we get the radial equation

$$
\frac{d^{2}}{d r^{* 2}} R+\left[\omega^{2}-\left(1-\frac{2 M}{r}\right)^{l(l+1)} \frac{r^{2}}{r}\right] R=0,
$$

where $d r^{*} \equiv(1-2 M / r)^{-1} d r$ or $r^{*}=r+2 M \ln (r / 2 M-1)$ + const introduces $r=r\left(r^{*}\right)$ as a function of the new variable $r^{*}$. Thus, the scattering of a vector E.M. wave by a Schwarzschild gravitational field ${ }^{14}$ behaves differently from that of a scalar wave obeying $\Psi^{; \mu} ; \mu=0$ whose radial part ${ }^{15}$ satisfies (10) with an additional term $-(1-2 M / r) 2 M / r^{3}$ in the square bracket.

\section{HIGH-FREQUENCY PROPAGATION IN WEAK SPHERICAL GRAVITATION}

\section{A. Approximate Equations}

Now, consider an E.M. wave in vacuum and of the form $\mathbf{E}(\mathbf{x}, t) \equiv \mathbf{f}(\mathbf{x}) e^{i[\Phi(x)-\omega t]}$, where $\mathbf{f}, \Phi$ are real functions, traveling in a star's external spherical gravity where $M / r \ll 1$. $^{16}$ For high-frequency propagation, we have

$$
\left|\frac{\left|\nabla^{2} \mathbf{f}\right|}{|\mathbf{f}|}\right|^{1 / 2}, \frac{|\boldsymbol{\nabla f}|}{|\mathbf{f}|}, \text { and } \frac{1}{r} \ll|\nabla \Phi|
$$

which physically means that $L(f) \gg L(\Phi)$ and $r \gg L(\Phi)$, where $L(f), L(\Phi)$ are, respectively, the typical distances over which f, $\Phi$ change significantly. Then for this wave and to the order $M / r$, (5) directly gives the amplitude-

\footnotetext{
${ }^{13}$ Ref. 8, Sec. 6B.

${ }^{14}$ T. C. Mo and C. H. Papas, Caltech Antenna Lab. report (unpublished).

${ }^{15}$ Ref. 6, Sec. II A.

${ }^{16}$ For example, $M_{\odot} / r \odot \sim 2.5 \times 10^{-6}, M \odot / R \oplus \sim 10^{-8}$, where $M \odot, r \odot$, and $R \oplus$ are the mass and radius of the sun, and the sun-earth distance, respectively. So even near the solar surface $r \sim r \odot$, the condition $M / r \ll 1$ is still satisfied. Except near the gravitational radius of very compact stars, such as a neutron star the condition $M / r \ll 1$ is always satisfied. Also, for the highfrequency E.M. propagation in a simple medium under uniform acceleration or in "uniform" gravity, see T. C. Mo, Radio Sci. (to be published).
}

propagation equation

$\boldsymbol{\nabla} \Phi \cdot \boldsymbol{\nabla} \mathbf{f}+\frac{1}{2}\left(\boldsymbol{\nabla}^{2} \Phi\right) \mathbf{f}=\frac{M}{r}\left[\frac{2 f^{(r)}}{r} \nabla \Phi+\Phi_{, r r} \mathbf{f}+2 \Phi_{, r} \mathbf{f}, r\right]$

and the well-known ${ }^{9}$ geometrical-optics eikonal equation

$$
(\nabla \Phi)^{2}-\frac{2 M}{r}\left(\frac{\partial \Phi}{\partial r}\right)^{2}=\left(1+\frac{2 M}{r}\right) \omega^{2} .
$$

To get the gravitational influence on the field amplitude, we simply insert into (12) the 3-dimensional phase information obtained from (13) and integrate along a ray path. Also, the presence of a simple material medium with very slowly changing $\mu$ and $\epsilon$ adds only a trivial factor $\mu \epsilon$ to the right-hand side of (13) and can be excluded.

\section{B. Amplitude Propagation}

The mathematics involved here is simplified if we introduce the harmonic coordinates ${ }^{17}\left\{t, r^{\prime} \equiv r-M, \theta, \phi\right\}$ in which (12) and (13) become

$$
\begin{gathered}
\boldsymbol{\nabla}^{\prime} \Phi \cdot \nabla^{\prime} \mathbf{f}+\frac{1}{2}\left(\nabla^{\prime 2} \Phi\right) \mathbf{f}=\frac{M}{r^{\prime 2}}\left[2 f^{(r)} \nabla^{\prime} \Phi-\mathrm{f} \frac{\partial \Phi}{\partial r^{\prime}}\right] \\
\left(\nabla^{\prime} \Phi\right)^{2}=\left(1+4 M / r^{\prime}\right) \omega^{2} \equiv n^{2} \omega^{2}
\end{gathered}
$$

We notice that from standard geometrical optics, ${ }^{18}\left(13^{\prime}\right)$ leads to the typical bending-ray paths $r^{\prime} \sin \theta=-2 M r^{\prime} / a^{\prime}$ $+a^{\prime}\left(1+2 M / a^{\prime}\right)$ and the phase velocity $\left(1+4 M / r^{\prime}\right)^{-1 / 2}$ along the ray. This immediately gives the familiar light deflection $^{1} \delta \theta=4 M / a^{\prime}$ and the radar traveling-time delay. ${ }^{2}$ Here $r^{\prime}=a^{\prime}, \theta=\frac{1}{2} \pi$ is the point of closest approach, where $d r^{\prime} / d \theta=0$. Also, the gravitational red shift is revealed by writing the proper frequency from the factor $e^{-i \omega t}$.

Now, the first-order $M / r^{\prime}$ perturbation solution of $\left(13^{\prime}\right)$ for a $z^{\prime}$-propagating unperturbed incident plane wave is

$$
\Phi=\omega\left[z^{\prime}+2 M \ln \left(r^{\prime}+z^{\prime}\right)+2 M g\left(x^{\prime}, y^{\prime}\right)\right] .
$$

Imposing the boundary condition that $\Phi$ at $z^{\prime}=-\infty$ represents a $z^{\prime}$-propagating incident plane wave, we determine $g\left(x^{\prime}, y^{\prime}\right)$ and get

$$
\begin{aligned}
& \nabla^{\prime} \Phi=\omega\left[\mathbf{e}_{z^{\prime}}\left(1+\frac{2 M}{r^{\prime}}\right)-\mathbf{e}_{\rho^{\prime}} \frac{2 M\left(r^{\prime}+z^{\prime}\right)}{r^{\prime} \rho^{\prime}}\right], \\
& \nabla^{\prime 2} \Phi \equiv 0,
\end{aligned}
$$

where $\rho^{\prime}$ and $\mathbf{e}_{\rho^{\prime}}$ are the polar radius and its direction in the $\left(x^{\prime}, y^{\prime}\right)$ plane. Equation (15) implies bending-ray paths $\left\{P: \quad r^{\prime} \sin \left(\theta+2 M / b^{\prime}\right)=-2 M r^{\prime} / b^{\prime}+b^{\prime}\right\} \quad$ along

${ }^{17}$ Ref. 9, pp. 193 and 215 .

${ }^{18}$ M. Klein, Electromagnetic Theory and Geometric Optics (Interscience, New York, 1965), pp. 64-74. Also see L. Landau and E. Lifshitz, Mechanics (Pergamon, New York, 1960), Chap. 1. 
which the field amplitudes are to be found. Here $\left\{b^{\prime}\right\}$, being the impact parameters of $\{P\}$, have the values of incidence heights $\rho^{\prime}$ at $z^{\prime}=-\infty$ as in Fig. 1. Equation (16) shows that the field lines of $\nabla^{\prime} \Phi$, which are identified as the rays, are conserved in the first-order gravitational bending.

Inserting (15) and (16) into (12') for a particular path $P$ in the $x^{\prime} z^{\prime}$ plane, we get the equation of $\mathbf{f}$ along $P$ :

$$
\begin{aligned}
\pm \frac{d}{d r^{\prime}} \mathbf{f}=\frac{M}{r^{\prime}\left(r^{\prime 2}+4\right.} & \left.M r^{\prime}-b^{\prime 2}\right)^{1 / 2} \\
\times & {\left[\mathbf{e}_{z} 2\left(f^{(x)} \frac{x^{\prime}}{r^{\prime}}+f^{(z)} \frac{z^{\prime}}{r^{\prime}}\right)-\mathbf{f} \frac{z^{\prime}}{r^{\prime}}\right], }
\end{aligned}
$$

where the path length $d \sigma^{\prime} \equiv \pm n r^{\prime}\left(n^{2} r^{\prime 2}-b^{\prime 2}\right)^{-1 / 2}$ is used, and,+- correspond to the two parts of the ray path after and before the point of closest approach, which occurs now at $r^{\prime}=a^{\prime}=b^{\prime}-2 M$. To order $M / r^{\prime}$ we can use the unperturbed straight path $\left\{P_{0}: \rho^{\prime}=b^{\prime}, z^{\prime}\right.$ $\left.= \pm\left(r^{\prime 2}-b^{\prime 2}\right)^{1 / 2}\right\}$ to integrate (17) and get the firstorder gravitational influence on amplitude propagation. We emphasize that the so-obtained results are valid only for $M / r^{\prime} \ll 1$ and only give a qualitative description when $M / \boldsymbol{r}^{\prime}$ approaches 1 .

(1) Radial propagation, $b^{\prime}=0$. Equation (17) directly gives

$$
\mathbf{f}=\left(f_{\infty}{ }^{(x)} e^{M / r^{\prime}}, f_{\infty}^{(y)} e^{M / r^{\prime}}, f_{\infty}^{(z)} e^{-M / r^{\prime}}\right),
$$

where $\mathbf{f}_{\infty}$ is the amplitude at $\boldsymbol{r}^{\prime}=\infty$. For the incident plane wave being considered $f_{\infty}(z) \equiv 0$, and the transverse field $f^{(t)}$ increases (decreases) from $f_{\infty}{ }^{(t)}$ at $r^{\prime}=\infty \quad\left(f_{a^{\prime}}{ }^{(t)}\right.$ at $\left.r^{\prime}=a^{\prime}\right)$ as $f_{\infty}{ }^{(t)} e^{M / r^{\prime}}\left(f_{a^{\prime}}(t) e^{\left.-M / a^{\prime}+M / r^{\prime}\right)}\right.$ when the wave propagates radially toward (away from) the star.

(2) Nonradial propagation, $b^{\prime} \neq 0$. For a chosen $P$ with given $b^{\prime}=a^{\prime}+2 M$, the results are most suitably expressed in the correspondingly rotated coordinates $\left\{x^{*}, y^{*} \equiv y^{\prime}, z^{*}\right\}$ which are obtained by rotating the coordinates $\left\{x^{\prime}, y^{\prime}, z^{\prime}\right\}$ about the $y^{\prime}$ axis through an angle $-2 M / a^{\prime}$ (Fig. 1). In this $\left\{x^{*}, y^{*}, z^{*}\right\}$, with its corresponding $P$ described by $x^{*}=-2 M r^{\prime} / a^{\prime}+a^{\prime}\left(1+2 M / a^{\prime}\right)$, (17) gives

$$
\begin{aligned}
& f^{\left(x^{*}\right)}=f_{a^{\prime}}\left(x^{*}\right) e^{\alpha\left(r^{\prime}\right)}, \\
& f^{\left(y^{*}\right)}=f_{a^{\prime}}\left(y^{*}\right) e^{\alpha\left(r^{\prime}\right)}, \\
& f^{\left(z^{*}\right)}=\left(2 M z^{*} / a^{\prime} r^{\prime}\right) f_{a^{\prime}}\left(x^{*}\right)
\end{aligned}
$$

where

$$
\alpha\left(r^{\prime}\right) \equiv \frac{M}{r^{\prime}}-\frac{M}{a^{\prime}}+\left[-\frac{2 M^{2}}{a^{\prime} r^{\prime}}+\frac{2 M^{2}}{a^{\prime 2}} \ln \left(1+\frac{a^{\prime}}{r^{\prime}}\right)\right]
$$

and $f_{a^{\prime}}{ }^{\left(x^{*}\right)}, f_{a^{\prime}}{ }^{\left(y^{*}\right)}$ are the field amplitudes at $r^{\prime}=a^{\prime}$. The square-bracketed quantity in (20) is written only to show that near $r^{\prime} \sim r_{1}^{\prime} \equiv a^{\prime 2} / 2 M$, where $P$ is bent to meet the $z^{*}$ axis and near which the straight-line $P_{0}$ integration starts breaking down. the first-order effect becomes

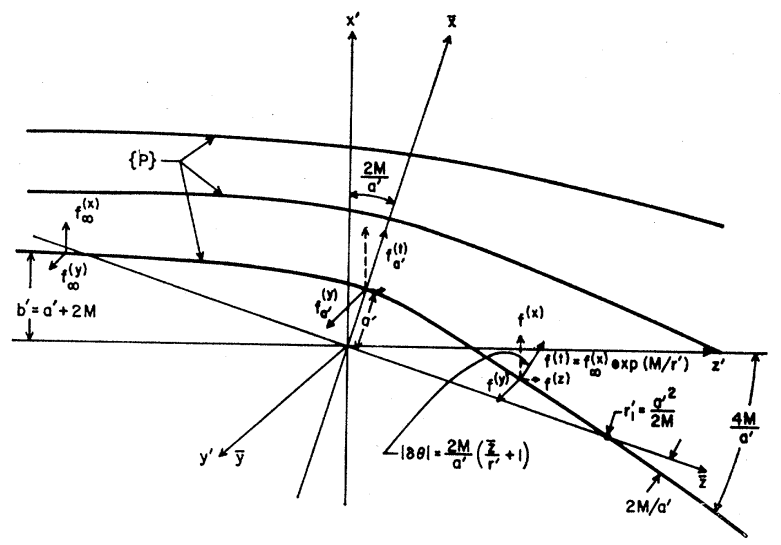

FIG. 1. High-frequency amplitude propagation in spherical gravity (The barred quantities are denoted by an asterisk in Sec. III B.)

as small as the second-order effect and thus both become negligible beyond $\boldsymbol{r}_{1}$.

From (19) the $f^{\left(z^{*}\right)}$ is coupled only to $f^{\left(x^{*}\right)}$ by just the right amount such that the total field on the $x^{*} z^{*}$ plane is bent through the angle $\delta \theta=2 M z^{*} / a^{\prime} r^{\prime}$ and is always kept perpendicular to $P$. Also to order $M / r^{\prime}$ both transverse fields, $f^{\left(y^{*}\right)}$ in the $y^{*}$ direction and $f^{\left(t^{*}\right)}$ on the $x^{*} z^{*}$ plane, are influenced equally. Thus the state of transverse polarization along propagation path is not affected by spherical gravity.

\section{Concluding Remarks}

Above we have investigated high-frequency planewave propagation. For a real transmitting antenna located in a gravitational field, we infer that a similar factor $e^{M / r^{\prime}}$ of gravitational perturbation modifies its high-frequency radiation field. Also because such an amplitude effect is not cumulative over the whole path $p$, as bending is, only receivers and transmitters at different radii can detect it. The maximum of this effect is $\delta f= \pm f_{a^{\prime}}\left(M / a^{\prime}\right)$ for a link from $r^{\prime}=a^{\prime}$ to $r^{\prime} \gtrsim r_{1}{ }^{\prime}$.

To measure such a small effect one needs in the first place highly sensitive receivers and transmitters. Also, for the round-trip amplitude effect not to cancel under the active-radar-transponding method, ${ }^{19}$ we could make the spacecraft near closest approach to a star $r^{\prime}=a^{\prime}$ transpond a signal inversely proportional to its received value. Then the earth station would receive twice the gravitational correction $\delta f_{r} / f_{r}=2 M / a^{\prime}$ to the amplitude or $\delta P_{r} / P_{r}=4 M / a^{\prime}$ to the power. For example, the $210-\mathrm{ft}$ antenna at Goldstone, Calif., can intercept $P_{r} \sim 10^{-18} P_{t}$ from such a transponder with power $P_{t}$, beam width $\sim 0.5^{\circ}$, at $\sim 1 \mathrm{AU}$ from earth and $\sim 1$ solar radius from the sun; then $\delta P_{r} / P_{r} \sim 10^{-6}$ or $\delta P_{r} \sim 10^{-24} P_{t}$ is the received power correction due to gravitation. It requires more than six-digits receiving-power precision at -240 $\mathrm{db}$ from the transponding power. This order of accuracy is not within the present capacity of the existing NASA

${ }^{19}$ V. C. Clarke, Jr., Jet Propulsion Lab. Report No. 605-504, 1970 (unpublished). 
Deep Space Instrumentation Facility. ${ }^{20}$ Nor can the passive radio-astronomical observations measure to such accuracy. ${ }^{21}$ But for an E.M. signal originating at

${ }^{20}$ Deep Space Instrumentation Facility, Jet Propulsion Lab System specification, Code No. 23835, Spec. No. DOW-1389-DTL, Rev. A., 1970 (unpublished).

${ }^{21}$ Typical signal-strength measurement is about to two-digit accuracy [A. T. Moffet (private communication)].
$M / r \lesssim 0.1$ or nearer to a compact star, such a change of signal strength should be observable on earth. In principle, this effect may provide another test of classical electromagnetic theory and general relativity, or may be used to single out the particular amplitude correction caused by gravitation.

\title{
Electron Scattering from a Standing Light Wave
}

\author{
R. Gush and H. P. Gush \\ Department of Physics, University of British Columbia, Vancouver, British Columbia, Canada
}

(Received 19 June 1970)

\begin{abstract}
The transition probability for electron scattering in the Kapitza-Dirac experiment is calculated using nonrelativistic theory as a function of the time of flight $t$ of the electron through the light beam, and as a function of the intensity. It is found that for intensities up to approximately $10^{7} \mathrm{~W} / \mathrm{cm}^{2}$ the probability equals $\sin ^{2} a_{0} t$, where $a_{0}$ is proportional to the intensity. At intensities of the order of $10^{9} \mathrm{~W} / \mathrm{cm}^{2}$, the dependence of the probability on the time is more complicated but it also oscillates between zero and nearly unity as the time increases. Both first- and second-order Bragg reflections are considered as well as conditions off the Bragg maximum.
\end{abstract}

\section{INTRODUCTION}

$I^{1}$ N 1933 Kapitza and Dirac ${ }^{1}$ predicted that electrons of well-defined momentum could be reflected from a standing light wave provided that $\lambda_{p}=\lambda \cos \vartheta$, where $\lambda_{p}$ is the de Broglie wavelength, $\lambda$ is the radiation wavelength, and $\vartheta$ is the angle of incidence. They called this a first-order Bragg condition, the lattice spacing being $\frac{1}{2} \lambda$. The probability per electron that reflection would take place was shown to be proportional to the square of the light intensity. At the time an experiment was impractical, given the intensity of available sources, but recently several attempts have been made to demonstrate this effect using $Q$-switched lasers as a light source. ${ }^{2}$ Electrons scattered by the light beam have been observed but the dependence of the probability of scattering on the angle of incidence and on the intensity of the light has not yet been determined experimentally.

The formula for the transition probability given by Kapitza and Dirac, ${ }^{1}$ derived using the theory of stimulated processes, is not valid at intensities used in current experiments, for it would predict probabilities in excess of unity. It is therefore essential to reexamine the theory of this scattering problem. The first extensive published investigation is that of Fedorov. ${ }^{3}$ The Schröd-

1 P. L. Kapitza and P. A. M. Dirac, Proc. Cambr. Phil. Soc. 29, 297 (1933).

${ }^{2}$ L. S. Bartell, R. R. Roskos, and H. Bradford Thompson, Phys. Rev. 166, 1494 (1968); L. S. Bartell, Phys. Letters 27A, 236 (1968); H.-Chr. Pfeiffer, ibid. 26A, 362 (1968); H. Schwarz, Ann. Physik 204, 276 (1967); Y. Takeda and I. Matsui, J. Phys. Soc. Japan 25, 1202 (1968).

${ }^{3}$ M. V. Fedorov, Zh. Eksperim. i Teor. Fiz. 52, 1434 (1967) [Sov. Phys. JETP 25, 952 (1967)]. inger equation, used to describe the electron, is cast into the form of a Mathieu equation by neglecting the time dependence of the standing light wave, treated as a classical field. Solutions are found, however, only in the case of either very low intensity, or of very high intensity, and predictions are not made in the intensity range used in recent experiments. Ezawa and Namaizawa ${ }^{4}$ have also investigated solutions to the Mathieu equation. Schoenebeck ${ }^{5}$ uses a modified first-order perturbation method to obtain a solution to the problem.

In this article a somewhat different approach is taken. The nonrelativistic Green function for an electron in a standing-wave field is calculated using perturbation theory. By neglecting the rapidly timevarying part of the electron-field interaction, it is possible to sum the perturbation series completely and obtain exact scattering matrix elements between states of definite electron momenta. This permits the transition probability to be evaluated for practically arbitrary intensities and interaction times. It is possible in addition to examine the transition probability for electron momenta not satisfying the Bragg condition, and to calculate the probability of higher-order Bragg reflections.

\section{THEORETICAL BACKGROUND}

\section{A. Preliminary Remarks}

The procedure adopted in this article is to calculate the scattering amplitude via the nonrelativistic Green

\footnotetext{
${ }^{4}$ H. Ezawa and H. Namaizawa, J. Phys. Soc. Japan 26, 458 (1969).

${ }^{5}$ H. Schoenebeck, Phys. Letters 27A, 286 (1968).
} 